\title{
Polifeprosan 20, 3.85\% carmustine slow release wafer in malignant glioma: patient selection and perspectives on a low-burden therapy
}

This article was published in the following Dove Press journal:

Patient Preference and Adherence

24 November 2016

Number of times this article has been viewed

\section{Lawrence Kleinberg}

Department of Radiation Oncology and Molecular Radiation Sciences, Johns Hopkins University, Baltimore, MD, USA
Correspondence: Lawrence Kleinberg Department of Radiation Oncology and Molecular Radiation Sciences, Johns Hopkins University, 40I North Broadway, Suite 1440, Baltimore, MD 2I23I, USA

Email kleinla@jhmi.edu

\begin{abstract}
Polifeprosan 20 with carmustine $\left(\right.$ GLIADEL $\left.^{\circledR}\right)$ polymer implant wafer is a biodegradable compound containing $3.85 \%$ carmustine (BCNU, bischloroethylnitrosourea) implanted in the brain at the time of planned tumor surgery, which then slowly degrades to release the BCNU chemotherapy directly into the brain thereby bypassing the blood-brain barrier. Carmustine implant wafers were demonstrated to improve survival in randomized placebo-controlled trials in patients undergoing a near total resection of newly diagnosed or recurrent malignant glioma. Based on these trials and other supporting data, carmustine wafer therapy was approved for use for newly diagnosed and recurrent malignant glioma in the United States and the European Union. Adverse events are uncommon, and as this therapy is placed at the time of surgery, it does not add to patient treatment burden. Nevertheless, this therapy appears to be underutilized. This article reviews the evidence for a favorable therapeutic ratio for the patient and the potential barriers. Consideration of these issues is important for optimal use of this therapeutic approach and may be important as this technology and other local therapies are further developed in the future.
\end{abstract}

Keywords: carmustine, wafer, gliadel, glioblastoma

\section{Introduction}

Despite intensive study directed at improving outcome, glioblastoma multiforme remains a universally fatal primary brain tumor with a median survival of $\sim 18$ months and rare survival beyond several years. Glioblastoma is a disease that is refractory to standard measures to obtain local control of the tumor, including maximal safe resection, partial brain radiotherapy administered to the tumor-bearing region, and chemotherapy. Essentially all patients will experience fatal progression within the brain despite this aggressive multimodality therapy. For $\sim 80 \%$ of patients, the initial recurrence is within $1 \mathrm{~cm}$ of the location of the initial tumor, thus motivating investigation of improved localized therapies. Patients may be under active treatment for a significant portion of their survival, including initial surgery, standard 6 weeks of concurrent chemoradiotherapy, and 6 months of standard adjuvant temozolomide chemotherapy, ultimately followed by combinations of additional surgery, radiation, and chemotherapy at the time of each treated recurrence. The recently US Food and Drug Administration (FDA)-approved electric "tumor-treating fields" therapy ${ }^{1}$ requires substantial effort with continuous use of a transducer helmet and battery pack from 1 month after radiotherapy until progression, with short interruptions for personal needs.

The blood-brain barrier appears to be an important obstacle to improving the therapeutic outcome as it limits penetration of most drugs and creates an immune-privileged 
space $^{2}$ that may limit effective antitumor response. Tight junctions are thought to selectively inhibit penetration of drugs with high molecular weight, negative charge, and low lipid solubility, and efflux pumps expel many drugs that do penetrate. ${ }^{2}$ Wafers (polifeprosan $20,3.85 \%$ carmustine) can be implanted (Figure 1) into the brain at the time of surgery, thereby overcoming the challenge of the blood-brain barrier by directly delivering chemotherapy by controlled release with no additional burden for the patient beyond the planned surgery. Carmustine wafers are efficacious with modest but meaningful benefit, having been demonstrated in randomized placebo-controlled trials to improve survival and quality of life outcome at initial therapy ${ }^{3,4}$ or recurrence. ${ }^{5}$ This therapy is only appropriate for a selected group of patients who are able to have a near total resection and may preclude subsequent eligibility for certain clinical trials, an issue of concern to patients and physicians seeking improved outcomes. Other approaches to increase drug delivery to the brain have not yet been proven to be efficacious and are generally associated with more risk, ${ }^{6,7}$ including convection-enhanced delivery using a catheter into the brain, direct injection, and osmotic disruption with or without intra-arterial drug injection.

Malignant glioma is a disease not only with a poor survival outcome but also with a clinical course that often substantially impacts quality of life and challenges caregivers, such that a treatment with modest risks requiring minimal effort is valued. Although a proportion of patients maintain a favorable performance status and independence through most of their survivorship, most do have periods during which they require substantial assistance with transportation for medical care as well as activities of daily living. Cognitive deficits may also have an impact on the ability to comply with treatment. Nevertheless, this therapy implanted during surgery with little additional risk or effort has not been widely adopted. Although overall usage statistics are not available, only $2.7 \%$ of patients

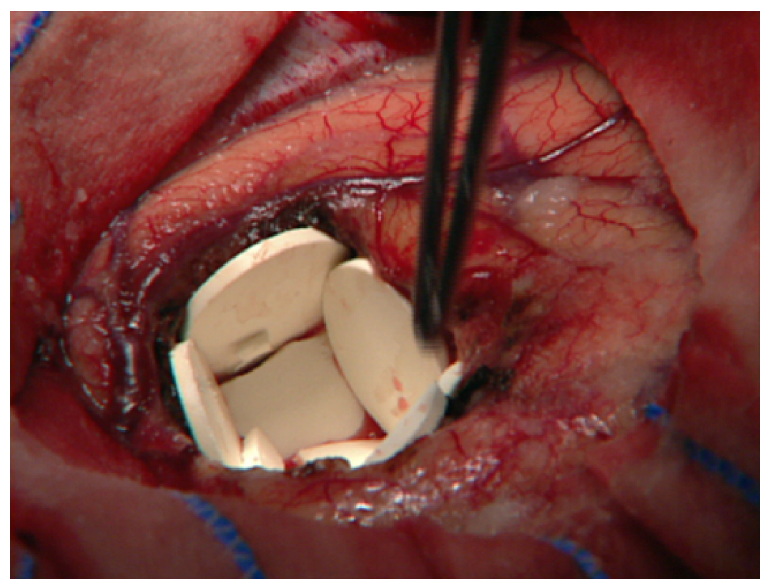

Figure I Carmustine wafers implanted in a glioblastoma resection cavity. in a recent international randomized trial that allowed eligibility after wafer implant ${ }^{1}$ had received carmustine wafers even though $64 \%$ of patients purportedly had a gross total complete resection and would have been candidates.

This review will explore the data most relevant to describing the benefits of this therapy in the context of minimal patient burden and risk, the rationale for and development of current concepts of optimal use in recurrent and newly diagnosed glioblastoma, and potential future opportunities to use this technology to improve outcomes.

\section{Background to therapeutic approach}

Polifeprosan 20, 3.85\% carmustine wafers (carmustine wafers) were developed as a means to deliver BCNU chemotherapy to glioblastoma patients by controlled release after implantation. ${ }^{8-11}$ This disease predominantly fails locally at or adjacent to the original tumor site. BCNU was selected as it was then used in standard management of malignant glioma. Although it was understood to penetrate the blood-brain barrier even when administered intravenously, increasing the concentration of this drug in the tumor by local delivery was an important goal, given its known activity in this disease. However, there may have been other interesting choices of drugs to administer using this technology, which are potentially active and yet have not been clinically useful as they do not otherwise penetrate the blood-brain barrier when systemically administered.

Although analysis of drug distribution in the human brain was not possible, pharmacokinetic studies in mammalian models suggest that the drug distribution goals are achieved. In a rabbit model, ${ }^{12} \sim 60 \%$ of $\mathrm{BCNU}$ was delivered $>3$ days after implant, whereas 7 days later very little could be detected. As predicted, the highest concentration is at the polymer/tissue interface falling off with depth into the brain. In a prior study, assessing drug distribution in rabbits, 3 days after implantation, ${ }^{13}$ meaningful concentrations of BCNU, defined as at least $10 \%$ of the concentration at the tissue/ polymer interface, were detected at a depth of 10-12 mm into the normal brain. In a primate study (cynomolgus monkeys), ${ }^{8,14}$ area under the curve (concentration over time) for drug in the brain was assessed as a metric for comparison with intravenous (IV) administration of BCNU. Standard IV administration was found to result in an area under the curve fourfold decrease in distant areas in the brain compared with wafer placement, and concentrations that were 25-1,200-fold less than those at the site of implantation.

Medical barriers to wide use of carmustine wafers include the need for careful selection of patients who will have a near total resection in a suitable anatomic location. Patient 
preference barriers include the need to provide informed consent for this therapy preoperatively for administration during surgery at the judgment of their physicians. For patients presenting for initial management, the discussion occurs at a time of stress without advance certainty of appropriateness, and in many cases even before the tumor diagnosis is even confirmed. Moreover, physicians must consider that wafer patients are not eligible for most clinical trials until the time of further tumor progression, creating the challenging dilemma of considering the tradeoff of omitting an FDA-approved therapy of meaningful but limited benefit for the speculative benefits of maintaining eligibility for clinical trials of unproven agents that the patient has not yet had the opportunity to explore. The reasons for exclusion of carmustine wafer patients from some investigational trials include possible synergistic risks, confounding of survival results, and difficulty assessing imaging. ${ }^{15,16}$ Such concerns about patient eligibility for future trials may also impact upon the physician team, thereby influencing patients. Finally, access may have been impeded in the United States as the medicare system did not provide hospitals with additional reimbursement for the cost of the carmustine wafers beyond standard payment for craniotomy until 2004, eight years after approval for routine clinical use.

More recently, temozolomide has been demonstrated to improve median and relatively longer term survival ${ }^{17,18}$ when combined with radiotherapy in the initial management of newly diagnosed patients. Although never compared with carmustine wafer-randomized trials, temozolomide chemotherapy results in a benefit in median survival that appears grossly similar (median survival improved by 2.2 months by carmustine wafer and 2.5 months by temozolomide) and a longer term benefit that may be modestly superior to placement of carmustine wafers (Table 1). Relative disadvantages of temozolomide include a 9-month treatment course and toxicities. Temozolomide chemotherapy had additional advantages including that the therapeutic decision occurs postoperatively after confirmation of pathology and is beneficial even for those who were unable to have a gross total resection. The additional value of using standard temozolomide when wafers have already been placed has not been well assessed, but data discussed below suggest that this is safe.

Testing for MGMT promotor methylation, an important prognostic factor for GBM in general even when treated with radiation alone and also predictive of benefit from temozolomide, ${ }^{18-20}$ was not available during the years of the prospective trial of carmustine wafer therapy and hence the potential advantage of carmustine wafer therapy as singleagent chemotherapy in the unmethylated temozolomide-resistant population remains unassessed. The unmethylated MGMT state has a worse prognosis for survival even for those who were treated with radiotherapy alone, and the available data suggest that outcome is worse for unmethylated rather than methylated patients receiving carmustine wafer therapy along with radiotherapy as expected. ${ }^{21-26}$ However, the available data are not sufficient to support any conclusions about whether use of carmustine wafers in the unmethylated population, more resistant to temozolomide, could be a superior choice. These and other identified predictive and prognostic markers may in the future guide personalized selection of therapies for high-grade glioma, including carmustine wafers. ${ }^{15}$

Table I Outcome of randomized trials assessing adjuvant use of carmustine implants or temozolomide compared with control adjuvant radiation arms

\begin{tabular}{|c|c|c|c|c|c|c|c|c|}
\hline \multirow{2}{*}{$\begin{array}{l}\text { Control arms } \\
\text { Study }\end{array}$} & \multicolumn{4}{|c|}{ Control arms (RT) } & \multicolumn{4}{|c|}{$\begin{array}{l}\text { Experimental arms (RT plus BCNU implant or RT } \\
\text { and temozolomide) }\end{array}$} \\
\hline & Number & $\begin{array}{l}\text { Median survival } \\
\text { (months) }\end{array}$ & $\begin{array}{l}\text { 2-year } \\
\text { survival (\%) }\end{array}$ & $\begin{array}{l}\text { 3-year } \\
\text { survival (\%) }\end{array}$ & Number & $\begin{array}{l}\text { Median } \\
\text { survival } \\
\text { (months) }\end{array}$ & $\begin{array}{l}\text { 2-year } \\
\text { survival (\%) }\end{array}$ & $\begin{array}{l}\text { 3-year } \\
\text { survival (\%) }\end{array}$ \\
\hline $\begin{array}{l}\text { Westphal et al } \\
\text { (control, placebo } \\
\text { wafer, and RT) })^{3, a}\end{array}$ & $\begin{array}{l}\text { I20 (placebo } \\
\text { wafer and RT) }\end{array}$ & 11.6 & 8.3 & 1.7 & $\begin{array}{l}\text { I20 (BCNU } \\
\text { wafer and RT) }\end{array}$ & 13.8 & 15.8 & 9 \\
\hline $\begin{array}{l}\text { EORTC: } \\
\text { (control, RT alone) })^{18,76}\end{array}$ & $\begin{array}{l}286(\mathrm{RT} \\
\text { alone) }\end{array}$ & 12.1 & 10.9 & 4.4 & $\begin{array}{l}287 \text { (RT and } \\
\text { TMZ) }\end{array}$ & 14.6 & 27 & 16 \\
\hline $\begin{array}{l}\text { EORTC: } \\
\text { biopsy only subgroup }\end{array}$ & 45 & 7.8 & 4.6 & 4.6 & 48 & 9.4 & 10.4 & 7.8 \\
\hline $\begin{array}{l}\text { EORTC: } \\
\text { partial resect subgroup }\end{array}$ & 128 & 11.7 & 9.4 & 3.7 & 126 & 13.5 & 23.7 & 14.3 \\
\hline $\begin{array}{l}\text { EORTC: complete } \\
\text { resect subgroup }\end{array}$ & 113 & 14.2 & 15.0 & 5.3 & 113 & 18.8 & 38.4 & 21.4 \\
\hline
\end{tabular}

Notes: Copyright @2012. Dove Medical Press. Reproduced from Kleinberg L. Polifeprosan 20, 3.85\% carmustine slow-release wafer in malignant glioma: evidence for role in era of standard adjuvant temozolomide. Core Evid. 2012;7:1 I5-130.16 a Results based on extent of resection not available for polymer study. Most would have had substantial or total debulking of gross disease based on intraoperative assessment.

Abbreviations: RT, radiotherapy; BCNU, carmustine; EORTC, European Organization for Research and Treatment of Cancer; TMZ, temozolomide; resect, resection. 


\section{Established role in recurrent high-grade glioma}

The initial evaluation of the utility of this novel therapeutic approach occurred in patients with recurrent tumor after initial treatment with radiotherapy and surgery, a situation for which there were no meaningfully effective nonsurgical options at the time. A Phase I trial for recurrent malignant glioma ${ }^{27}$ patients undergoing resection proceeded through three BCNU wafer concentration escalation steps of $1.93 \%$, $3.85 \%$, and $6.35 \%$ with median survival after implant of 65 weeks, 64 weeks, and 32 weeks, respectively. Based on this information, the $3.85 \%$ loading dose was selected for development, despite a decision based on this Phase I study that was not designed to reliably assess survival outcome. In fact, there was an imbalance toward a higher proportion of confirmed GBM in the highest dose level, which may be the cause of poorer survival. The therapy is tolerable, and even at the highest dose level there was not sufficient systemic concentration of BCNU to cause any toxic events.

A blinded, placebo-controlled Phase III trial followed ${ }^{5}$ to assess the value of this therapy for recurrent high-grade glioma. A total of 222 patients were randomly assigned to have implantation during planned surgical resection of biodegradable Polifeprosan 20 polymer disks with or without $3.85 \%$ carmustine incorporated. Eligibility included otherwise recommended surgical resection of a single unilateral contrast enhancing recurrent high-grade glioma $>1 \mathrm{~cm}$ in size and Karnofsky Performance Status $\geq 60$. Approximately $80 \%$ of enrolled patients had a $>75 \%$ resection of tumor. A total of $65 \%$ had glioblastoma as final pathology at the time of reoperation, whereas $18 \%$ had anaplastic Grade III tumors and $14 \%$ had other glial tumors, highlighting the challenge of using intraoperative preliminary pathology assessment in guiding therapy.

The results suggested a short-term survival benefit that was within the range achieved for many therapies generally accepted for use in advanced malignant cancers. Adjusting for prognostic factors, median survival of the carmustine wafer treated significantly better at 31 weeks, whereas it was 23 weeks for patients treated with placebo polymers (hazard ratio $=0.67, P=0.006$ ). However, the benefit did not meet the criteria for statistical significance on the prespecified unadjusted analysis. Among patients with confirmed glioblastoma (Grade IV) at the time of this repeat surgery, 6-month survival of those treated with active wafers was meaningfully greater (64\% vs $44 \%, P=0.02$ ). Neither systemic nor intracranial toxicity appeared elevated. Although the primary end point of survival benefit in unadjusted analysis of all randomized patients was not met, these benefits were considered meaningful, and US FDA approval was granted in 1996 for this indication and in 2002 in the European Union.

The decision that was made by the drug development team to pursue $3.85 \%$ loading based on limited survival data from a small Phase I study may have resulted in a missed opportunity for a more efficacious treatment and is instructional. The maximum tolerated concentration of BCNU loaded in the wafer was evaluated in a multi-institutional NCI-funded New Approaches to Brain Tumor Therapy Consortium dose escalation trial. ${ }^{28}$ Polymer loading with $\mathrm{BCNU}$ was escalated through $6.5 \%, 10 \%, 14.5 \%, 20 \%$, and $28 \%$ BCNU. The $20 \%$ loading was selected for further study as at the $28 \%$ loading three of four patients had significant edema or wound complications. A cohort of 20 patients treated with the $20 \%$ loading confirmed this as a safe dosing. Low concentrations of BCNU in serum were detectible, but the concentrations were $\sim 500$ times lower than concentrations demonstrated to cause systemic toxicity. Unfortunately, although a larger trial was considered to determine whether there was a superior survival benefit with this loading, $5.2 \times$ more than the commercially available standard, the outcome with this higher carmustine concentration was ultimately never studied.

\section{Role in initial therapy}

The first advance in standard management of malignant glioma results from a practice-changing randomized trial published in $1978,29,30$ which demonstrated that a 6-week course of daily radiotherapy treatments improved the median survival of high-grade glioma from 14 weeks to 35 weeks even though survival outcome beyond 2 years remained quite uncommon. An $\sim 6$-week course of radiotherapy is utilized to minimize the risk of injury that may result from higher daily doses, although shorter courses are justifiable in poor prognosis circumstances. Until that trial was completed, there was controversy about whether the burden of this prolonged course of treatment in setting of short survival was worthwhile. These studies also suggested that adjuvant intravenous BCNU chemotherapy, suspected to be active for glioma ${ }^{31,32}$ and to cross the blood-brain barrier, may improve the possibility of relatively long-term survival for 1-2 years beyond the benefit of radiotherapy alone. Notably, this benefit (the "tail on the curve") from this chemotherapy regimen did not translate into better very long-term survival nor did it achieve statistical significance.

The therapy next shown to improve survival beyond the effects of radiotherapy in initial management of glioblastoma was actually carmustine wafers, published 25 years later 
in 2003. ${ }^{3,4}$ A 22-patient Phase I trial confirmed the safety of radiotherapy along with carmustine wafers. ${ }^{33}$ A Phase III randomized trial intended to definitively assess this therapy was initiated in Norway and Finland ${ }^{34}$ but aborted when there was an interruption in drug supply. The results were analyzed for the 32 patients actually enrolled, which demonstrated that median survival was increased from 40 weeks to 58 weeks $(P=0.012)$. Although there was an imbalance resulting in the presence of a higher proportion of more favorable Grade III histology patients in the carmustine wafer arm, analysis of the GBM subgroup suggested the possibility of a meaningful improvement in median survival from 40 weeks to 53 weeks $(P=0.008)$.

Motivated by these findings, the manufacturer sponsored a more definitive 230 patient-blinded placebo-controlled trial. ${ }^{3,4}$ A statistically significant survival benefit, improved from 11.6 months to 13.9 months, was observed. The hazard rate for death was reduced by $29 \%(P=0.03)$. A total of $80 \%$ of patients had glioblastoma, as only frozen section pathology was available at the time of implantation. When the GBM subgroup was analyzed, the median survival was improved from 11.4 months to 13.5 months, which was not statistically significant. However, when other potential prognostic factors were accounted for using a Cox proportional hazards model, a significant $31 \%$ reduction in the risk of death $(P=0.04)$ was observed, leading to approval for this indication by the US FDA in 2003 and the European Union in 2004. The British $\mathrm{NHS}^{35}$ as part of its evaluation of this therapeutic approach requested an unplanned subgroup analysis including the additional variable of extent of resection as the known limited depth of BCNU penetration raised questions about appropriateness for patients with residual bulk disease. Interestingly, the survival benefit was only significant in the population with $>90 \%$ resection of gross contrast-enhancing disease. For this subgroup, there was an improvement in mean and median survival of 4.2 months and 2.15 months, respectively $(P=0.0061)$, which is considered meaningful for this illness.

As radiation therapy has a significant impact on brain imaging, progression-free survival is not a useful end point for treatment success. Progression-fee survival was a secondary end point for the randomized trial for newly diagnosed patients. Progression, determined based on radiographic (25\% increase in the largest cross-sectional area or a new lesion) or clinical criteria, was 5.9 months for both arms. This caused concern about whether the treatment was actually beneficial. However, since then it had become clear that imaging progression after radiation treatment in the brain is not a useful end point as the effects of radiation may mimic actual progression (called pseudopression). ${ }^{36,37}$ Out of a group of 45 patients treated with radiotherapy and carmustine wafers, ${ }^{38}$ five of 15 patients (33\%, encompassing $11 \%$ of all treated patients) selected for reoperation for resectable recurrence after carmustine wafers and radiotherapy actually had pure treatment effect or necrosis with no active glioma when the specimens were examined. Over time, greater information has been describing imaging findings, which may occur after carmustine implantation with or without concurrent radiotherapy that provides guidance. ${ }^{39-41}$ With this knowledge, caution is recommended in determining recurrence after brain radiotherapy, regardless of the use of wafers, and it is generally good practice to alert patients treated with brain radiotherapy that later signs of progression on imaging may not be accurate.

Toxicity and neurologic adverse events were similar on both arms and therefore are unlikely to have been a barrier to more widespread use of this therapy. Neurologic adverse events including seizures, neurologic deficits, and operative complications were similar in both groups. In this study, cerebrospinal fluid (CSF) leak was $5 \%$ vs $0.8 \%$ without increase in infections. Subsequent reports do not confirm a high rate of CSF leak. ${ }^{42}$ A meta-analysis ${ }^{15}$ of published data reports $<1 \%$ fatal complications possible related to carmustine wafers, and a $<1 \%$ need to remove the wafers for possible adverse effects.

These toxicity outcome data are from randomized trials where both the BCNU group and the placebo group had wafers implanted, but did not contain groups with craniotomy alone. Therefore, it remained important to consider whether there could be increased complications resulting from the blank placebo wafers, which might have interfered with detection of increased treatment-related events. Retrospective data suggest that there is no increased risk related to polymer placement itself. For example, a large single institution report from Johns Hopkins ${ }^{42}$ reported operative complications in 288 patients receiving carmustine implant (166 newly diagnosed, 122 for recurrence) in comparison with 725 patients having craniotomy without any polymer for malignant glioma. Patients in carmustine implants vs craniotomy cohorts had similar incidences of perioperative surgical site infection $(2.8 \%$ vs $1.8 \%, P=0.33)$, CSF leak $(2.8 \%$ vs $1.8 \%, P=0.33)$, meningitis $(0.3 \%$ vs $3 \%, P=1.00)$, incisional wound-healing difficulty $(0.7 \%$ vs $4 \%, P=0.63)$, symptomatic malignant edema $(2.1 \%$ vs $2.3 \%, P=1.00)$, 3 -month seizure incidence ( $14.6 \%$ vs $15.7 \%$, $P=0.65)$, deep-vein thrombosis $(6.3 \%$ vs $5.2 \%, P=0.53)$, 
and pulmonary embolism $(4.9 \%$ vs $3.7 \%, P=0.41)$. For the complications of wound healing and infection, repeat surgery for recurrence is associated with increased risk of infection but not use of carmustine wafers. ${ }^{43}$ There has not been observation of hematologic toxicity potentially related to $\mathrm{BCNU}$ released from the wafers in any clinical context.

Giese et $\mathrm{al}^{44}$ proposed strategies to reduce complications based on the early experience with polymer implants, emphasizing the following techniques: 1) perioperative anticonvulsants and dexamethasone; 2) watertight dural closure; 3) limit potential for contamination of dural closure by BCNU by irrigation and not using instruments with prior contact with BCNU for dural closure; and 4) prophylactic intraoperative and postoperative antibiotics. In addition, a significant connection between the surgical cavity and the ventricular system has long been considered to create a risk of obstructive hydrocephalus should a polymer or polymer fragment enter the CSF space.

The impact of this therapy on quality of life appeared appropriate for a treatment in an illness that remained fatal in the relatively short term for most patients. Although rigorous quality of life instruments were not utilized, the study suggested a symptom or quality of life benefit ${ }^{3}$ to the therapy, and the toxicity data did not suggest consequent events that might negatively impact quality of life. The primary functional end point was decline in performance status, and there was significant improvement with use of wafers in median time to decline from 10.4 months to 11.9 months with 1 -year deterioration-free rate of $48 \%$ vs $39 \%(P=0.05)$. Moreover, a statistically significant benefit was also demonstrated for ten of eleven other individual function and neurologic examination elements assessed.

As a point of comparison related to assessment of treatment burden vs benefit, the Brain Tumor Study Group randomized trial reported in $1978^{29,30}$ confirmed the value of radiotherapy in improving median survival and also demonstrated that the addition of intravenous administration of carmustine did not improve median survival, but did lead to a statistically nonsignificant improvement in 1 year and 18 months survival. This small benefit led to frequent use as a standard option in the United States even though 2-year survival remained negligible. In contrast, carmustine wafers have been demonstrated to have a survival benefit extending several years for some patients, while only uncommonly resulting in significant toxicity. Systemic administration of BCNU chemotherapy, commonly used based on the Brain Tumor Study Group data, results in a significant risk of thrombocytopenia, $<90,000$ in $~ 25 \%$ of patients and $<50,000$ in $6 \%-7 \%$ along with a seemingly less substantial survival benefit.
Adjuvant temozolomide chemotherapy is now standardly used in the therapy of Grade III and IV malignant glioma. Temozolomide was approved for use in the United States in newly diagnosed glioblastoma in 2005. The routine use of temozolomide chemotherapy, ${ }^{17,18}$ demonstrated to improve median and 3- to 5-year survival, has necessitated re-evaluation of optimal use of carmustine implants for newly diagnosed patients. Temozolomide is an oral drug taken every day during radiotherapy, and then for six 5 -day monthly cycles. Frequent blood tests are required to monitor for thrombocytopenia. It is also utilized at the time of recurrence after initial therapy. A meta-analysis of studies ${ }^{45}$ assessing benefits of carmustine wafers including randomized trials and cohort studies suggested an HR for survival of 0.59 (95\% CI 0.44-0.79), which was similar to the benefit to temozolomide of 0.63 (95\% CI, 0.52-0.75) in the trial reported by Stupp et al. ${ }^{18}$

The current optimal role for carmustine wafer implants at the time of initial surgery is more controversial in the absence of randomized data or large prospective series utilizing carmustine implants, radiotherapy, and temozolomide in combination. First of all, it is unknown whether systemic temozolomide chemotherapy or local carmustine wafer implant leads to meaningfully improved survival compared with the other approach when used in appropriately selected patients. Limited evidence exists from several small studies that carmustine implants used at the time of initial resection are safe in this new clinical context, where temozolomide is to be given along with and subsequent to radiotherapy, and at least raise the question of whether the addition of carmustine wafer may improve the outcome of temozolomide with radiotherapy..$^{8,12,15,16,22-26,46-58}$ Indeed, the lack of prospective data from comparative trials about outcome for a large cohort of patients also may reduce patient and physician enthusiasm for the use of carmustine wafer implants in the initial management of newly presenting glioblastoma. Selection factors, patient heterogeneity, and the limitations on benefits of any therapy compound the difficulties in guiding patients based on the available data. In our practice, we do continue to offer carmustine wafers as a choice for selected surgically resectable and eligible patients after careful discussion of the alternatives.

\section{Wafer technology: possible renewed interest in the future?}

The development of the carmustine wafer provides proof of principle that this technology, and perhaps other approaches to local drug delivery, can improve survival outcome for high-grade glioma. This technology is particularly attractive 
for patients as it is completed at the time of surgery without need for implanted devices, externalized catheters, or extended hospital stays. There are several clear opportunities to improve this therapy, although unfortunately enthusiasm for investment and grant funding may have been dampened by limited diffusion of the existing technology into routine practice.

As discussed earlier, a clinical trial ${ }^{28}$ has demonstrated that wafers with a higher concentration of BCNU could be safely utilized potentially to improve drug distribution and consequently outcome. There may also be a greater benefit in exploring the use of other chemotherapy agents with this delivery system. BCNU was selected as the agent as it had known activity in malignant glioma. Interestingly, IV BCNU was likely active in part because it crosses the blood-brain barrier, a factor which may decrease usefulness of local administration as it can also easily exit via the same route. ${ }^{8,59}$ For example, when inulin, a large molecule that has less potential to cross the blood-brain barrier, is administered by wafer technology, the inulin persists in the brain for a longer period of time and diffuses a larger distance from the brain/polymer interface. The feasibility of polifeprosan 20 polymer as a slow release delivery technology for alternative therapeutic agents has been demonstrated in preclinical models using paclitaxel, ${ }^{60}$ IUDR, ${ }^{61,62}$ temozolomide, ${ }^{63}$ taxotere ${ }^{64}$ camptothecin, ${ }^{65,66}$ tiripazamine, ${ }^{67}$ and other agents. When more effective agents are identified, this may be considered again. For example, polymer has been used ${ }^{68}$ to improve outcome with human GBM in a mouse model by delivering at a high concentration Axl small molecule tyrosine kinase inhibitor with a greater range of diffusion than $\mathrm{BCNU}$, which impacts migration and invasion.

Interestingly, the development of lymphocyte-mediated immune therapy using checkpoint inhibitors to treat tumors may provide motivation to reassess the potential benefits of carmustine wafers. Profound- and prolonged treatmentrelated lymphopenia with reduced CD4 counts has been demonstrated in malignant glioma after radiotherapy with or without temozolomide or BCNU, ${ }^{69-71}$ and this may be associated with reduced tumor-related survival. This phenomenon has been observed in lung, ${ }^{72}$ pancreas, ${ }^{73}$ and head and neck tumors ${ }^{74}$ as well. There is concern that this phenomenon may limit the effectiveness of new lymphocyte-mediated immune therapies. Drug delivery by this polymer technology may be an effective way to deliver otherwise myelosuppressive chemotherapy without further impacting immune response. When utilized in a murine GBM model ${ }^{75}$ in combination with systemic anti-Pd1 monoclonal antibody (anti-PD1ma) therapy, local delivery of BCNU resulted in significantly better survival and a more robust immune response than observed with systemic BCNU concurrent with anti-PD1ma therapy or either therapy alone. Moreover, durable immune response was confirmed by failure of reimplantation in longterm survivors only after local injection and anti-PD1ma and not after other combinations. A clinical trial to assess this in human patients is under development.

\section{Conclusion}

The challenges of the development and utilization of carmustine wafers may provide interesting lessons. The technology was shown to improve survival, with little risk and almost no patient effort thus it would be expected to have been widely accepted by patients and physicians. Although exact statistics are not available, it has apparently been utilized for only a modest proportion of eligible patients. Although the benefits are controversial for newly diagnosed patients in the current era when temozolomide is routinely used, the approach appears appropriate for selected patients who are able to undergo a near total resection. The advantages and disadvantages of using this as a low-burden alternative to temozolomide for select populations remain untested. Carmustine implant is clearly appropriate for those who have recurred after prior therapy but are still suitable for a near total resection, even though most will have received prior therapy with the alkylating agent temozolomide. Future opportunities may result from personalized patient selection and potentially by utilizing this technology to deliver other agents or use of carmustine wafers in combination with immune therapies.

As this and other similar technologies may be further developed in the future, consideration of the barriers that were encountered may be important. Possibilities include 1) ineligibility for experimental studies, 2) need for rigorous surgical technique, 3) most institutions may not encounter enough eligible patients to warrant stocking the product, 4) need to discuss and obtain consent from patients even before tumor is confirmed in a setting where it may preclude later options, 5) modest survival improvement similar to many oncology drugs but perhaps not customarily sufficient for the neurosurgical community to alter practice, 6) physician biases. Consideration and study of these issues is important to facilitate diffusion of this and related approaches into practice, and 7) delayed creation of a mechanism for payment of the additional cost beyond standard craniotomy alone.

\section{Disclosure}

The authors report no conflicts of interest in this work. 


\section{References}

1. Stupp R, Taillibert S, Kanner AA, et al. Maintenance therapy with tumortreating fields plus temozolomide vs temozolomide alone for glioblastoma: a randomized clinical trial. JAMA. 2015;314(23):2535-2543.

2. Pitz MW, Desai A, Grossman SA, Blakeley JO. Tissue concentration of systemically administered antineoplastic agents in human brain tumors. J Neurooncol. 2011;104(3):629-638.

3. Westphal M, Hilt DC, Bortey E, et al. A phase 3 trial of local chemotherapy with biodegradable carmustine (BCNU) wafers (Gliadel wafers) in patients with primary malignant glioma. Neuro Oncol. 2003;5(2): 79-88.

4. Westphal M, Ram Z, Riddle V, Hilt D, Bortey E; Executive Committee of the Gliadel Study Group. Gliadel wafer in initial surgery for malignant glioma: long-term follow-up of a multicenter controlled trial. Acta Neurochir (Wien). 2006;148(3):269-275. [discussion 275].

5. Brem H, Piantadosi S, Burger PC, et al. Placebo-controlled trial of safety and efficacy of intraoperative controlled delivery by biodegradable polymers of chemotherapy for recurrent gliomas. The Polymer-brain Tumor Treatment Group. Lancet. 1995;345(8956):1008-1012.

6. Mehta AI, Linninger A, Lesniak MS, Engelhard HH. Current status of intratumoral therapy for glioblastoma. J Neurooncol. 2015;125(1): $1-7$.

7. Doolittle ND, Muldoon LL, Culp AY, Neuwelt EA. Delivery of chemotherapeutics across the blood-brain barrier: challenges and advances. Adv Pharmacol. 2014;71:203-243.

8. Fleming AB, Saltzman WM. Pharmacokinetics of the carmustine implant. Clin Pharmacokinet. 2002;41(6):403-419.

9. Domb AJ, Israel ZH, Elmalak O, Teomim D, Bentolila A. Preparation and characterization of carmustine loaded polyanhydride wafers for treating brain tumors. Pharm Res. 1999;16(5):762-765.

10. Domb AJ, Rock M, Schwartz J, et al. Metabolic disposition and elimination studies of a radiolabelled biodegradable polymeric implant in the rat brain. Biomaterials. 1994;15(9):681-688.

11. Domb A. Gliadel - a preparation for the supplementary treatment of brain cancer. Harefuah. 1999;137(3-4):127-131.

12. Domb AJ, Rock M, Perkin C, Yipchuck G, Broxup B, Villemure JG. Excretion of a radiolabelled anticancer biodegradable polymeric implant from the rabbit brain. Biomaterials. 1995;16(14):1069-1072.

13. Grossman SA, Reinhard C, Colvin OM, et al. The intracerebral distribution of BCNU delivered by surgically implanted biodegradable polymers. J Neurosurg. 1992;76(4):640-647.

14. Fung LK, Ewend MG, Sills A, et al. Pharmacokinetics of interstitial delivery of carmustine, 4-hydroperoxycyclophosphamide, and paclitaxel from a biodegradable polymer implant in the monkey brain. Cancer Res. 1998;58(4):672-684.

15. Chowdhary SA, Ryken T, Newton HB. Survival outcomes and safety of carmustine wafers in the treatment of high-grade gliomas: a metaanalysis. J Neurooncol. 2015;122(2):367-382.

16. Kleinberg L. Polifeprosan 20,3.85\% carmustine slow-release wafer in malignant glioma: evidence for role in era of standard adjuvant temozolomide. Core Evid. 2012;7:115-130.

17. Stupp R, Mason WP, van den Bent MJ, et al; European Organisation for Research and Treatment of Cancer Brain Tumor and Radiotherapy Groups; National Cancer Institute of Canada Clinical Trials Group. Radiotherapy plus concomitant and adjuvant temozolomide for glioblastoma. $N$ Engl J Med. 2005;352(10):987-996.

18. Stupp R, Hegi ME, Mason WP, et al. Effects of radiotherapy with concomitant and adjuvant temozolomide versus radiotherapy alone on survival in glioblastoma in a randomised phase III study: 5-year analysis of the EORTC-NCIC trial. Lancet Oncol. 2009;10(5):459-466.

19. Mirimanoff RO, Gorlia T, Mason W, et al. Radiotherapy and temozolomide for newly diagnosed glioblastoma: recursive partitioning analysis of the EORTC 26981/22981-NCIC CE3 phase III randomized trial. J Clin Oncol. 2006;24(16):2563-2569.

20. Hegi ME, Diserens AC, Gorlia T, et al. MGMT gene silencing and benefit from temozolomide in glioblastoma. $N$ Engl J Med. 2005;352(10): 997-1003.
21. Grossman R, Burger P, Soudry E, et al. MGMT inactivation and clinical response in newly diagnosed GBM patients treated with Gliadel. J Clin Neurosci. 2015;22(12):1938-1942.

22. Noel G, Schott R, Froelich S, et al. Retrospective comparison of chemoradiotherapy followed by adjuvant chemotherapy, with or without prior gliadel implantation (carmustine) after initial surgery in patients with newly diagnosed high-grade gliomas. Int J Radiat Oncol Biol Phys. 2012; 82(2):749-755

23. Bock HC, Puchner MJ, Lohmann F, et al. First-line treatment of malignant glioma with carmustine implants followed by concomitant radiochemotherapy: a multicenter experience. Neurosurg Rev. 2010; 33(4):441-449.

24. Salmaggi A, Milanesi I, Silvani A, et al. Prospective study of carmustine wafers in combination with 6-month metronomic temozolomide and radiation therapy in newly diagnosed glioblastoma: preliminary results. J Neurosurg. 2013;118(4):821-829.

25. Gutenberg A, Bock HC, Bruck W, et al. MGMT promoter methylation status and prognosis of patients with primary or recurrent glioblastoma treated with carmustine wafers. Br J Neurosurg. 2013;27(6):772-778.

26. Lechapt-Zalcman E, Levallet G, Dugue AE, et al. O(6)-methylguanineDNA methyltransferase (MGMT) promoter methylation and low MGMT-encoded protein expression as prognostic markers in glioblastoma patients treated with biodegradable carmustine wafer implants after initial surgery followed by radiotherapy with concomitant and adjuvant temozolomide. Cancer. 2012;118(18):4545-4554.

27. Brem H, Mahaley MS Jr, Vick NA, et al. Interstitial chemotherapy with drug polymer implants for the treatment of recurrent gliomas. J Neurosurg. 1991;74(3):441-446.

28. Olivi A, Grossman SA, Tatter S, et al; New Approaches to Brain Tumor Therapy CNS Consortium. Dose escalation of carmustine in surgically implanted polymers in patients with recurrent malignant glioma: a new approaches to brain tumor therapy CNS consortium trial. J Clin Oncol. 2003;21(9):1845-1849.

29. Walker MD, Alexander E Jr, Hunt WE, et al. Evaluation of BCNU and/or radiotherapy in the treatment of anaplastic gliomas. A cooperative clinical trial. J Neurosurg. 1978;49(3):333-343.

30. Walker MD, Green SB, Byar DP, et al. Randomized comparisons of radiotherapy and nitrosoureas for the treatment of malignant glioma after surgery. N Engl J Med. 1980;303(23):1323-1329.

31. Burger PC. Malignant astrocytic neoplasms: classification, pathologic anatomy, and response to treatment. Semin Oncol. 1986;13(1):16-26.

32. Nieder C, Grosu AL, Molls M. A comparison of treatment results for recurrent malignant gliomas. Cancer Treat Rev. 2000;26(6): 397-409.

33. Brem H, Ewend MG, Piantadosi S, Greenhoot J, Burger PC, Sisti M. The safety of interstitial chemotherapy with BCNU-loaded polymer followed by radiation therapy in the treatment of newly diagnosed malignant gliomas: phase I trial. J Neurooncol. 1995;26(2):111-123.

34. Valtonen S, Timonen U, Toivanen P, et al. Interstitial chemotherapy with carmustine-loaded polymers for high-grade gliomas: a randomized double-blind study. Neurosurgery. 1997;41(1):44-48. [discussion 48-49].

35. NHS: National Institute for Health and Clinical Excellence [webpage on the Internet]. Carmustine implants and temozolomide for the treatment of newly diagnsoed high-grade glioma. Available from: www.nice.org. uk/TA121. Accessed July 29, 2016.

36. Brandes AA, Tosoni A, Spagnolli F, et al. Disease progression or pseudoprogression after concomitant radiochemotherapy treatment: pitfalls in neurooncology. Neuro Oncol. 2008;10(3):361-367.

37. Chamberlain MC. Pseudoprogression in glioblastoma. J Clin Oncol. 2008;26(26):4359. [author reply 4359-4360].

38. Kleinberg LR, Weingart J, Burger P, et al. Clinical course and pathologic findings after Gliadel and radiotherapy for newly diagnosed malignant glioma: implications for patient management. Cancer Invest. 2004; 22(1):1-9.

39. Colen RR, Zinn PO, Hazany S, et al. Magnetic resonance imaging appearance and changes on intracavitary Gliadel wafer placement: a pilot study. World J Radiol. 2011;3(11):266-272. 
40. Hammoud DA, Belden CJ, Ho AC, et al. The surgical bed after BCNU polymer wafer placement for recurrent glioma: serial assessment on $\mathrm{CT}$ and MR imaging. AJR Am J Roentgenol. 2003;180(5):1469-1475.

41. Masuda Y, Ishikawa E, Yamamoto T, et al. Early postoperative expansion of parenchymal high-intensity areas on $\mathrm{T} 2$-weighted imaging predicts delayed cerebral edema caused by carmustine wafer implantation in patients with high-grade glioma. Magn Reson Med Sci. Epub 2015 Dec 28.

42. Attenello FJ, Mukherjee D, Datoo G, et al. Use of Gliadel (BCNU) wafer in the surgical treatment of malignant glioma: a 10-year institutional experience. Ann Surg Oncol. 2008;15(10):2887-2893.

43. Chaichana KL, Kone L, Bettegowda C, et al. Risk of surgical site infection in 401 consecutive patients with glioblastoma with and without carmustine wafer implantation. Neurol Res. 2015;37(8):717-726.

44. Giese A, Bock HC, Kantelhardt SR, Rohde V. Risk management in the treatment of malignant gliomas with BCNU wafer implants. Cen Eur Neurosurg. 2010;71(4):199-206.

45. Xing WK, Shao C, Qi ZY, Yang C, Wang Z. The role of Gliadel wafers in the treatment of newly diagnosed GBM: a meta-analysis. Drug Des Devel Ther. 2015;9:3341-3348.

46. Salvati M, D'elia A, Frati A, Brogna C, Santoro A, Delfini R. Safety and feasibility of the adjunct of local chemotherapy with biodegradable carmustine (BCNU) wafers to the standard multimodal approach to high grade gliomas at first diagnosis. J Neurosurg Sci. 2011;55(1):1-6.

47. Salmaggi A, Duri S, Silvani A, et al. Loco-regional treatments in firstdiagnosis glioblastoma: literature review on association between Stupp protocol and Gliadel. Neurol Sci. 2011;32(suppl 2):S241-S245.

48. Perry J, Chambers A, Spithoff K, Laperriere N. Gliadel wafers in the treatment of malignant glioma: a systematic review. Curr Oncol. 2007;14(5): 189-194.

49. Pan E, Mitchell SB, Tsai JS. A retrospective study of the safety of BCNU wafers with concurrent temozolomide and radiotherapy and adjuvant temozolomide for newly diagnosed glioblastoma patients J Neurooncol. 2008;88(3):353-357.

50. Menei P, Metellus P, Parot-Schinkel E, et al; Neuro-oncology Club of the French Society of Neurosurgery. Biodegradable carmustine wafers (Gliadel) alone or in combination with chemoradiotherapy: the French experience. Ann Surg Oncol. 2010;17(7):1740-1746.

51. McGirt MJ, Than KD, Weingart JD, et al. Gliadel (BCNU) wafer plus concomitant temozolomide therapy after primary resection of glioblastoma multiforme. J Neurosurg. 2009;110(3):583-588.

52. McGirt MJ, Brem H. Carmustine wafers (Gliadel) plus concomitant temozolomide therapy after resection of malignant astrocytoma: growing evidence for safety and efficacy. Ann Surg Oncol. 2010;17(7):1729-1731.

53. Affronti ML, Heery CR, Herndon JE 2nd, et al. Overall survival of newly diagnosed glioblastoma patients receiving carmustine wafers followed by radiation and concurrent temozolomide plus rotational multiagent chemotherapy. Cancer. 2009;115(15):3501-3511.

54. Dixit S, Hingorani M, Achawal S, Scott I. Retrospective comparison of chemoradiotherapy followed by adjuvant chemotherapy, with or without previous gliadel implantation (carmustine) after initial surgery in patients with newly diagnosed high-grade gliomas: in regard to Noel et al. (Int J Radiat Oncol Biol Phys. 2011. DOI:10.1016/j. ijrobp.2010.11.073). Int J Radiat Oncol Biol Phys. 2011;81(5):1593.

55. Gopferich A. Erosion of composite polymer matrices. Biomaterials. 1997;18(5):397-403.

56. La Rocca RV, Mehdorn HM. Localized BCNU chemotherapy and the multimodal management of malignant glioma. Curr Med Res Opin. 2009;25(1):149-160.

57. Duntze J, Litre CF, Eap C, et al. Implanted carmustine wafers followed by concomitant radiochemotherapy to treat newly diagnosed malignant gliomas: prospective, observational, multicenter study on 92 cases. Ann Surg Oncol. 2013;20(6):2065-2072.

58. Pallud J, Audureau E, Noel G, et al; Club de Neuro-Oncologie of the Société Française de Neurochirurgie. Long-term results of carmustine wafer implantation for newly diagnosed glioblastomas: a controlled propensity-matched analysis of a French multicenter cohort. Neuro Oncol. 2015;17(12):1609-1619.
59. Arifin DY, Lee KY, Wang CH, Smith KA. Role of convective flow in carmustine delivery to a brain tumor. Pharm Res. 2009;26(10): 2289-2302.

60. Walter KA, Cahan MA, Gur A, et al. Interstitial taxol delivered from a biodegradable polymer implant against experimental malignant glioma. Cancer Res. 1994;54(8):2207-2212.

61. Williams JA, Dillehay LE, Tabassi K, Sipos E, Fahlman C, Brem H. Implantable biodegradable polymers for IUdR radiosensitization of experimental human malignant glioma. J Neurooncol. 1997;32(3): 181-192.

62. Yuan X, Dillehay LE, Williams JR, Williams JA. Synthetic, implantable polymers for IUdR radiosensitization of experimental human malignant glioma. Cancer Biother Radiopharm. 1999;14(3):187-202.

63. Brem S, Tyler B, Li K, et al. Local delivery of temozolomide by biodegradable polymers is superior to oral administration in a rodent glioma model. Cancer Chemother Pharmacol. 2007;60(5):643-650.

64. Sampath P, Rhines LD, DiMeco F, Tyler BM, Park MC, Brem H. Interstitial docetaxel (taxotere), carmustine and combined interstitial therapy: a novel treatment for experimental malignant glioma. $J \mathrm{Neu}-$ rooncol. 2006;80(1):9-17.

65. Storm PB, Moriarity JL, Tyler B, Burger PC, Brem H, Weingart J. Polymer delivery of camptothecin against 9L gliosarcoma: release, distribution, and efficacy. J Neurooncol. 2002;56(3):209-217.

66. Weingart JD, Thompson RC, Tyler B, Colvin OM, Brem H. Local delivery of the topoisomerase I inhibitor camptothecin sodium prolongs survival in the rat intracranial 9L gliosarcoma model. Int J Cancer. 1995; 62(5):605-609.

67. Yuan X, Tabassi K, Williams JA. Implantable polymers for tirapazamine treatments of experimental intracranial malignant glioma. Radiat Oncol Investig. 1999;7(4):218-230.

68. Yen SY, Chen SR, Hsieh J, et al. Biodegradable interstitial release polymer loading a novel small molecule targeting Axl receptor tyrosine kinase and reducing brain tumour migration and invasion. Oncogene. 2016;35(17):2156-2165.

69. Yovino S, Kleinberg L, Grossman SA, Narayanan M, Ford E. The etiology of treatment-related lymphopenia in patients with malignant gliomas: modeling radiation dose to circulating lymphocytes explains clinical observations and suggests methods of modifying the impact of radiation on immune cells. Cancer Invest. 2013;31(2):140-144.

70. Kleinberg L, Grossman SA, Piantadosi S, Zeltzman M, Wharam M. The effects of sequential versus concurrent chemotherapy and radiotherapy on survival and toxicity in patients with newly diagnosed high-grade astrocytoma. Int J Radiat Oncol Biol Phys. 1999;44(3):535-543.

71. Grossman SA, Ye X, Lesser G, et al; NABTT CNS Consortium. Immunosuppression in patients with high-grade gliomas treated with radiation and temozolomide. Clin Cancer Res. 2011;17(16):5473-5480.

72. Campian JL, Ye X, Brock M, Grossman SA. Treatment-related lymphopenia in patients with stage III non-small-cell lung cancer. Cancer Invest. 2013;31(3):183-188.

73. Wild AT, Herman JM, Dholakia AS, et al. Lymphocyte-sparing effect of stereotactic body radiation therapy in patients with unresectable pancreatic cancer. Int J Radiat Oncol Biol Phys. 2016;94(3):571-579.

74. Campian JL, Sarai G, Ye X, Marur S, Grossman SA. Association between severe treatment-related lymphopenia and progression-free survival in patients with newly diagnosed squamous cell head and neck cancer. Head Neck. 2014;36(12):1747-1753.

75. Mathios D, Phallen J, Park C, et al. PD-1 blockade shows synergistic survival, anti-tumor immune response and long-term memory with interstitial but not systemic chemotherapy: study in a murine glioblastoma model. Neuro Oncol. 2014;16:110.

76. Stupp R, Gander M, Leyvraz S, Newlands E. Current and future developments in the use of temozolomide for the treatment of brain tumours. Lancet Oncol. 2001;2(9):552-560. 


\section{Publish your work in this journal}

Patient Preference and Adherence is an international, peer-reviewed, open access journal that focuses on the growing importance of patient preference and adherence throughout the therapeutic continuum. Patient satisfaction, acceptability, quality of life, compliance, persistence and their role in developing new therapeutic modalities and compounds to optimize

clinical outcomes for existing disease states are major areas of interest for the journal. This journal has been accepted for indexing on PubMed Central. The manuscript management system is completely online and includes a very quick and fair peer-review system, which is all easy to use. Visit http://www. dovepress.com/testimonials.php to read real quotes from published authors.

Submit your manuscript here: http://www.dovepress.com/patient-preference-and-adherence-journal 